\title{
Wireless Smart Node and Private Cloud Based Environmental Noise Monitoring System
}

\author{
Song Hao \\ College of Mechanical \& Electrical Engineering,North China University of Technology Beijing, China
}

\begin{abstract}
This paper introduces a wireless smart node and private cloud based city ambient noise KEYWORDS detection and processing system. With the function of noise data preprocessing and storage, Wireless sensor network self-diagnostics, and power management, the smart node implements a high-precision, Prvite cloud wide-area and continuous noise data acquisition in urban environment. Data are send back from smart node all-round the city to processing center running on private cloud Environmental noise monitoring Data fusion through public mobile communication networks. Applications on the private cloud manage, analyzing and do a data fusion job with geographic information system. The system requires less setting and maintenance cost, and has more flexibility for update and extension.
\end{abstract}

\section{Introduction}

Along with economic growth and Urbanization, people pay more and more attention on life quality include environment they lived in. City ambient noise pollution as well as air quality and water pollution is an extremely urgent problem. Differ from air or water pollution, source of noise has the character of wide distributed, more liquidity and shorter duration time so that hard to detection and processing. Traditional noise monitoring system needs lot of human resource but get few noise data useful for back-end analysis.

On the other way, developing of computer and information technology makes "Cyber Earth" and "Smart City" from concept to reality. Mobile networks and "cloud" give a new path for environmental noise signal detection, transport and processing. Wireless smart node and private cloud based environmental noise monitoring system includes a series of distributed detection node to acquire on field noise signal data and do some processing like saving and compressing before the data are send back to data center though public mobile communication networks. A private cloud is set up for time and position based noise data storage and querying. Applications running on the cloud can evaluate intensity and frequency of the noise data, do a data fusion from noise source with geographic information system. All the results obtained above provide scientific basis for city noise control projects and making of developing plan.

Copyright (C) 2016 Song Hao

doi: $10.18686 /$ esta.v3i1.9

This is an open-access article distributed under the terms of the Creative Commons Attribution Unported License (http://creativecommons.org/licenses/by-nc/4.0/), which permits unrestricted use, distribution, and reproduction in any medium, provided the original work is properly cited.

${ }^{*}$ Corresponding author: Song Hao Email:songhao@ncut.edu.cn
The on field smart node has a outstanding performance omnidirectional and professional microphone which complies with the IEC61672 class 2 sound level meter standard, and a ARM Architecture based low-power consumption center processing unit(CPU) for environmental noise detection and transport. The private is made up of a net work gateway, storage server and kinds of data processing applications. More details will be introduced in the following chapters.

\section{On Field Smart Node for Noise Detection}

\section{Constitution of the smart node}

PThe main modules of the node are:

1. A IEC61672 class 2 sound level meter standard complied professional microphone.

2. A high performance embedded system with a ARM CPU used for noise data detection, preprocessing the data before transport.

3. Solar- lithium battery and it's management circuit as power supply system.

Structure diagram of the noise detection smart node is shown in Fig. 1.

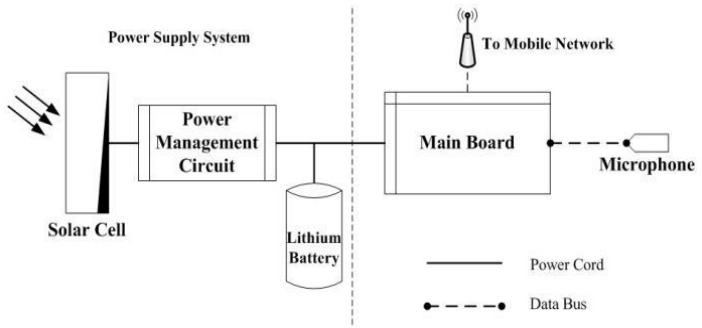

Fig. 1. Smart node hardware components. 


\section{The microphone}

A pre-calibrated pressure condenser microphone which has a nearly perfect circle of directional performance under the frequency of $1 \mathrm{KHz}$ and a flat frequency response curve under $2 \mathrm{KHz}$ is used in the smart node for noise signal detection. The microphone is competent for city environmental noise detection which mainly happens at low frequencies and from all directions. Directional characteristic of the microphone is shown in Fig. 2.

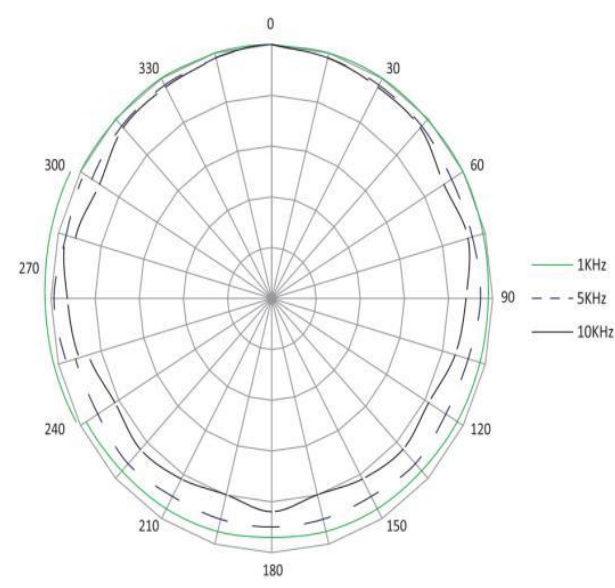

Fig. 2. Omni-directional chart of the microphone

The typical frequency response curve at the distance of $100 \mathrm{~cm}$ from noise source is shown in Fig. 3.

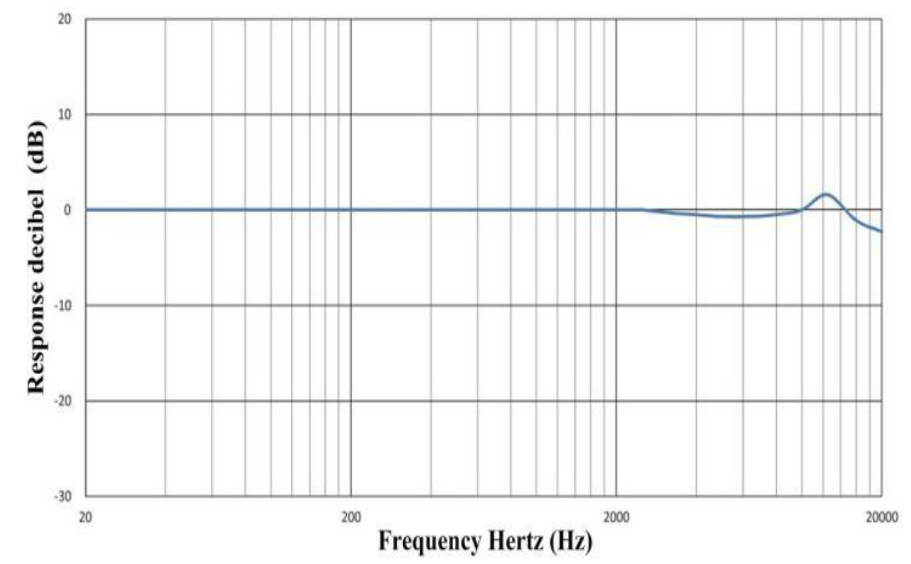

Fig. 3. Frequency response of the microphone

\section{Other Hardware of The Node}

The smart noise detection node is consist of solar battery based power supply module, main board with ARM CPU, global positioning system (GPS) module and communication module

1) Solar- lithium battery and it's management circuit

The distributed noise detection node use a solar-lithium battery system for less difficulty of setting up and maintenance. Solar cell panel in the system has max power output of more than 5 watt, and the lithium battery is at least 0.5 ampere hour at 3.7 volt output. The solar panel charges the lithium battery though a power management circuit, and it's needs less than a day fully charging the battery. The power management circuit has the function of battery health monitoring and will give a alarm signal to main board if the solar panel give nothing output up to more than 24 hours. Then the main board turns the system working in a power-saving mode such as using a lower sampling rate, sending fewer data back to data center and sends error message back. Node with a fully charged battery can work at least 100 hours in power saving mode and do a continuously monitoring before repair.

2) High performance ARM CPU based main board

ARM A7 or A15 processor which have the character of outstanding compute capacity and low energy consumption is used in the noise detection node for high sampling rate environmental noise detection and data pre-processing and system working state monitoring. There is build-in flash memory on board for temporally data storage.

3) GPS and communication module

Noise data can be attached with time and position information from GPS module, and the information is very useful for data fusion with geographic information system to generate city noise map and make city developing plan.

Node uses the 3rd or 4th generation mobile communication network for noise data transfer, and there is enough bandwidth (usually up to 1 mage-bit per second) for continuously noise data and control signal real time transport.

\section{Software of The Node}

Software running on the node main board do the job of noise signal detection, pre-processing ( such as filtering and compressing ), data saving, communicating and working state monitoring. The software can adjust working mode according to the instructions from data center, and working as pre-set mode when the net link is broken.

\section{Private Cloud}

On field environmental noise data are transferred through mobile communication networks to a gate way before being saved in the storage server running on the private cloud. Then the data will be processed by on-cloud applications for analysis and data fusion.

\section{Communication Network and Gateway}

On field noise data and node working state information delivered on the 3rd or 4th generation mobile communication networks should enter the system gateway. The gateway keeps the rules to access the private cloud by port control, media access control, and device number management for data safety.

\section{The Private Cloud}

All the noise storage management and data processing work are finished by applications on the private cloud. Not like public cloud system, the noise monitoring system's private cloud is connected to Internet with the special gateway. so that the private cloud system has the superiority of data integrity, accessing stability and expansion flexibility.

Structure diagram of the private cloud for city environmental noise monitoring system is shown in Fig. 4. 


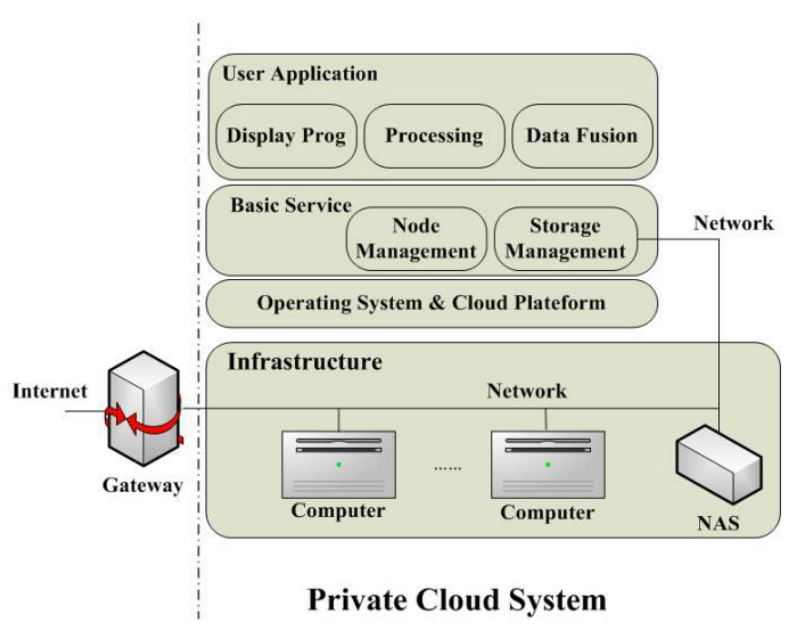

Fig. 4. Structure diagram of the private cloud

As presented in Fig. 4, main software module running on the private cloud is:

1) Node working state monitoring, management and transfer control module

Software managements all the information of the smart noise detection node such as node position and device number, get the node self monitoring data and highlight the failure message. The node's working state like sampling rate and communication mode can be remote controlled by the software on cloud.

2) Environmental noise data management module

This application saves all data from on field detection node into the database on cloud, and regularly backs up the data to a Network Attached Storage ( NAS ) for data safety. The data management module also gives a database interface for all the noise data processing applications on cloud to read, write and query the data.

3) Noise data processing module

Noise data processing module includes a series of applications on environmental noise monitoring system private cloud who's tasks are mainly all the data send back in real-time and classification mode, to analysis and do data fusion with geographic information system for city noise map and city developing plan.

Applications and programs in the processing module can be either public analysis software or customized application for special use on the city noise monitoring system. All these programs and applications are on the cloud, any of the authorized computer can access the full function software while do not need install them.

\section{Advantages of the System}

Compare to the traditional city noise detection system, the wireless smart node and private cloud based environmental noise monitoring system has some peculiar advantages from using the wireless smart node and private cloud. Some of the most important superiority with this system are list as follow:

\section{Wireless Network, Easy for setup and}

The smart on field noise detection node uses wireless mobile communication network for date transfer, avoids difficulty of wire routing and save much of manual work. Noise detection node can be mounted most efficiency place for comprehensive and accuracy monitoring. Other type of sensor such as air quality or temperature can easily be installed onto the smart node so that the system will be converted to a city environmental Integrated monitoring system.

\section{Software on cloud, easy for update}

All the noise data processing applications are on the private cloud, that's a solution of "one time install, always to use". Computers authorized can access applications without install them. Once the applications updated from cloud, all users get new software function automatically.

\section{Flat and Robust Monitoring Network, Low Cost}

Smart noise detection nodes has the same priority, work separately in the system and establish directly communication with back-end data center for data transfer. Some nodes failure have no effect to others. Each node which has its storage recourse and a solarlithium battery as power supply can work a rather long time when power failure or network broken, thus save some cost for system maintenance.

\section{Rich Data for Intelligent City}

All data Noise send back to data center are attached with time and position information from GPS module, which is very useful for data fusion with geographic information system to generate city noise map, noise control project and city developing plan.

\section{Conclusion}

With wireless mobile communication networks and private cloud, the city environmental noise monitoring system designed smart noise detection nodes that can automatically acquire noise data, monitor working state and communicate with back end data center. Applications on private cloud management, save and process the date for data fusion.

The system get rich data with lower cost, represents the intelligent city's development direction.

\section{Acknowledgment}

Author Song Hao thanks my wife for years of supporting in both everyday life and working.

Thanks to College of Mechanical \& Electrical Engineering, North China University of Technology for research environment.

\section{References}

1. ZHU Na, LIU Lin, "Wireless Sensor Networks," Value Engineering, vol. 33, pp.222-223, October 2014.

2. Ren Fengyuan,Huang Haining,Lin Chuang, "Wireless Sensor Networks," Journal of Software, vol. 14, pp.1282-1291, July 2003.

3. MELL P,GRANCE T, The NIST definition of cloud computing, Gaithersburg, NIST, 2012.

4. WWW.MIC-W.COM, "i series kit user manual 2012," unpublished.

5. Boss G, Malladi P, Quan D, Legregni L, Hall H, Cloud computing. IBM White Paper, http://download.boulder.ibm.com/ $\mathrm{ibmdl} / \mathrm{pub} / \mathrm{software} / \mathrm{dw} / \mathrm{wes} /$ hipods/Cloud computing wp final 8Oct.pd, 2007.

6. Amazon, Amazon elastic compute cloud (Amazon EC2), http://aws.amazon.com/ec2/, 2009.

7. Rings T,Grabowski J,Schuiz S, “ Grid and cloud computing:opportunities for integration with the next generation network ," Journal of Grid, vol. 3, pp. 375393, July, 2009.

8. Wind S, "Open Source Cloud Computing Management Platforms Introduction, Comparison, and Recommendations for Implemen- tation , 2011 IEE[Conference on Open Systems (ICOS 2011) , pp.175-179, September 2011. 